\title{
Quantum dots impair macrophagic morphology and the ability of phagocytosis by inhibiting the Rho-associated kinase signaling
}

\author{
Guangbo Qu, ${ }^{a}$ Changwen Zhang, ${ }^{a}$ Lin Yuan, ${ }^{a}$ Jiuyang He, ${ }^{a b}$ Zhe Wang, ${ }^{a}$ Lixin Wang, ${ }^{a}$ Sijin Liu ${ }^{* a}$ \\ and Guibin Jiang ${ }^{a}$
}

Received 31st January 2012, Accepted 16th February 2012

DOI: $10.1039 / \mathrm{c} 2 \mathrm{nr} 30243 \mathrm{~h}$

\begin{abstract}
Quantum dots (QDs) are fluorescent semiconductor nanoparticles that have broad excitation spectra, narrow emission peaks, long fluorescence lifetimes, and the ability to easily conjugate with bio-molecules. Due to these distinct characteristics, QDs represent promising substances in biological imaging and labelling. However, the side and adverse effects of QDs are also widely studied. Herein, we recognize macrophages as the pivotal cells in ingesting QDs, and that the accumulation of QDs inside macrophages leads to significant morphological alterations and a remarkable reduction of their ability to erythrophagocytize in vitro. In a mouse model with chronic exposure to QDs, red blood cell (RBC) retention in spleens and severe splenomegaly were observed, presumably due to attenuated macrophagic erythrophagocytosis in vivo. Importantly, we demonstrated that QDs greatly inhibited the Rho-associated kinase (ROCK) activity, resulting in impaired fidelity of the actin cytoskeleton and actin-rich structure (such as surface protrusions), which was assumed to be the molecular basis underlying the blunted macrophagic morphology and reduced ability to phagocytize. The combined data provide insights into QDs' intracellular trafficking, localization and biological fate in macrophages, and the resultant impairment to cytoskeleton coupled with inhibition on the ROCK signalling would decrease the macrophagic ability to erythrophagocytize with diminished $\mathrm{RBC}$ recycling and splenic $R B C$ retention in animals.
\end{abstract}

\section{Introduction}

The application of nanotechnology in the diagnosis and therapy for various diseases has recently attracted significant attention. Nanoparticles reveal promising applications in medical practice, such as imaging, diagnosing, drug delivery vehicles and anti-cancer drugs. Among them, quantum dots (QDs) are unique photoable nanoparticle probes possessing novel characteristics in fluorescence, such as narrow emission peaks and wide excitation spectra. ${ }^{1}$

${ }^{a}$ State Key Laboratory of Environmental Chemistry and Ecotoxicology, Research Center for Eco-Environmental Sciences, Chinese Academy of Sciences, Beijing 100085, China.E-mail: sjliu@rcees.ac.cn

${ }^{b}$ College of Biological Science and Technology, Jinan University, Guangzhou 510070, China
QD-fluorescence was first used to detect F-actin in mouse fibroblasts. ${ }^{2}$ Since then, numerous studies have been carried out to assess their application in cell and animal imaging for cancer invasiveness, lymphocyte homing and embryogenesis, and data from the current literature recognized QDs as potent fluorescent probes for in vivo imaging of lymph nodes and tumors. ${ }^{3}$ Additionally, QDs can be readily conjugated to different bio-molecules (e.g. antibodies) for site-directed delivery or targeting. These together endow QDs with beneficial features in imaging and potentially in site-directed drug delivery.

Whilst human beings can benefit from nano-products, potential toxicities to human health upon exposure to nanomaterials from the routes of daily use and medical therapeutics are of significant concern to the public. It is therefore important to gain adequate insights into the uptake, trafficking, clearance and biological influences of nanomaterials in order to eliminate their potential adverse and side effects. A large sum of studies have documented the toxicities of various nanoparticles, such as oxidative stress and inflammation upon exposure to QDs, nanosilver and carbon nanotubes. ${ }^{4}$ In light of the toxicity of QDs, a number of studies have shown their adverse influence on a wide spectrum of cells. ${ }^{5}$ Our previous results demonstrated that the liver is the preferential and predominant organ for QD accumulation. ${ }^{6}$ When mice were exposed to chronic and acute QD treatment, the hepatic deposition of QD particles resulted in significant impairment to lobular morphology, and caused dramatic oxidative stress. ${ }^{6}$ However, we still do not know whether there is also damage to Kuppfer cells, a cardinal type of macrophages in the liver, and whether macrophages take the principal role in holding QDs. Herein, we therefore delineate the engulfing of QDs by macrophages, and the causal influence on macrophage-mediated erythrophagocytosis. The combined results from the current study suggest that QD exposure leads to impairments not only in macrophagic morphology but also its biological function.

\section{Materials and methods}

Nanoparticles and characterization

The CdSe QD stock solution was stored in the dark at $4{ }^{\circ} \mathrm{C}$. The CdSe QD particles pre-coated with polyethylene glycol (PEG) were purchased from Wuhan Jiayuan Quantum Dots Co., LTD., China. The nanomaterials were characterized prior to experiments. The 
morphologies of QDs were assessed by transmission electron microscopy (TEM, Hitachi H-7500, Japan), and the particle sizes were assayed using a nanoparticle size analyzer (N5, Backman, USA). The shape and morphology of the QDs were similar to those described in our recent study, ${ }^{6}$ and the diameter of the QDs was about $4 \mathrm{~nm}$. The fluorescence spectrum measured by a Hitachi F-4500 spectrofluorometer (Hitachi Co. Ltd., Tokyo, Japan) indicated that the maximum emission wavelength was $590 \pm 10 \mathrm{~nm}$, and the maximum half width was $\leq 32 \mathrm{~nm}$. For animal and cell treatments, CdSe QD nanoparticles were spun down and then re-dissolved in PBS. The control animals or cells received PBS only.

\section{Animal experimentation}

All mouse breeding and experiments were approved by the Committee of Animal Care at the RCEES, Chinese Academy of Sciences. Regarding the chronic QD exposure, mice were administrated with $200 \mathrm{nM}$ CdSe QDs in $100 \mu \mathrm{l}$ PBS via tail vein (305.6 $\mathrm{ng} \mathrm{kg}^{-1}$ body weight) every other day for 12 days. The mice were sacrificed $24 \mathrm{~h}$ after the final administration, and spleen and liver samples were collected. The control mice received PBS only. Histological examination was performed as discussed previously. ${ }^{7}$

\section{Cell culture}

Mouse monocyte-macrophage J774A.1 cells and mouse hepatocytes Hepa 1-6 cells (both purchased from the Shanghai Cell Bank of Type Culture Collection of CAS) were cultured in DMEM and 1640 medium (Hyclone), respectively, supplemented with 10\% fetal bovine serum (Gibco) and $100 \mathrm{U} \mathrm{mL}^{-1}$ penicillin/streptomycin (Gibco). Cell experiments were carried out similarly to those described in our recent publication. ${ }^{6}$ Nanoparticles were spun down and then re-dissolved in PBS prior to cell treatments. The control cells received PBS only.

\section{Cell viability assay}

J774A.1 and Hepa 1-6 cells were seeded in 24 well plates with $1 \times 10^{5}$, and starved in medium with $1 \%$ serum for $12 \mathrm{~h}$. Then, cells were treated with QDs at 20 and $100 \mathrm{nM}$. After $24 \mathrm{~h}$ and $48 \mathrm{~h}$, cell counting was performed with a hemocytometer.

\section{Co-culture of hepatocytes and macrophages}

J774A.1 and Hepa 1-6 cells were detached, centrifuged at $3000 \mathrm{~g}$ and then re-suspended with DMEM and 1640 medium, respectively. After cell counting, both types of cells were diluted to $5 \times 10^{5}$ cells $\mathrm{ml}^{-1}$, and the J774A.1 cell suspension was added into the Hepa $1-6$ suspension $(1: 1, \mathrm{v} / \mathrm{v})$. The mixed cell suspension was seeded in 6-well plates. One day after incubation, $20 \mathrm{nM}$ QDs were exposed to the co-cultured cells for $24 \mathrm{~h}$. The QD fluorescence was assessed under a fluorescent microscope.

\section{Confocal laser scanning microscopy}

After incubation with $20 \mathrm{nM}$ QDs for 24 h, J774A.1 cells were fixed with $10 \%$ PBS-buffered formaldehyde and washed with $0.1 \%$ Triton $\mathrm{X}-100$ in PBS for $10 \mathrm{~min}$. The cell nuclei were stained with DAPI (blue), and F-actin in the cytoskeleton was stained with FITC-conjugated phalloidin (green), as previously described. ${ }^{8}$
Confocal laser scanning microscopy was then performed to visualize fluorescent images following the instructions as described previously. ${ }^{9}$

\section{Erythrophagocytosis by macrophages}

Erythrophagocytosis was performed as previously described. ${ }^{10}$ Briefly, senescence of RBCs was induced by increasing intracellular calcium concentration with $\mathrm{CaCl}_{2}$ and $\mathrm{Ca}^{+2}$ ionophore $\mathrm{A} 23187$ (Sigma). Senescent RBCs were incubated with J774A.1 macrophages with or without QD treatment $\left(3 \times 10^{7} \mathrm{RBCs} /\right.$ well in 24-well plates). Non-ingested RBCs were eliminated with the RBC lysis reagent. Cells were checked and visualized by phase-contrast microscopy, and macrophages with ingested RBCs were quantified.

\section{ROCK kinase activity assay}

After washing twice with ice-cold PBS, an equal number of cells were lysed with the RIPA lysis buffer from Pierce, USA. The ROCK kinase activity was assayed with a kit from Millipore, USA, following the manufacturer's instructions.

\section{Western blot analysis}

Cells were lysed using the RIPA lysis buffer from Pierce. An equal amount of protein extract from each group was loaded for separation by $4-12 \%$ SDS-PAGE. Western blot analysis was performed following a standard protocol. The antibodies against GAPDH $(1: 1000)$ and the anti-phosphorylated MLC $(1: 1000)$ were both purchased from Santa Cruz Biotechnology, USA. GAPDH was used as a loading control.

\section{Statistical analysis}

The difference of experimental data between two groups was assessed using the two-tailed Student's $t$-test. $P<0.05$ was considered statistically significant. All results were presented in mean \pm SD.

\section{Results and discussion}

Although our previous results substantiated the liver as the prominent site in mice for the localization of QDs, we still lack insight into the differential distribution of QDs between hepatocytes and macrophages (Kuppfer cells). In other words, whether or not Kuppfer cells take the foremost role in ingesting QDs due to their ability to engulf foreign particles is not established. QDs could not be seen in the liver slides after the fixative treatment from mice with QD exposure (data not shown). ${ }^{6}$ We thus circumvented this issue by employing a co-culture system of two types of cells, ${ }^{11}$ hepatocytes and macrophages, which are two major types of cells in the liver. In the co-culture system, murine hepatocytes Hepa 1-6 and macrophages J774A. 1 cells were treated with $20 \mathrm{nM}$ QDs, at which cell viability for both Hepa 1-6 and J774A.1 were not significantly altered for $24 \mathrm{~h}$ and $48 \mathrm{~h}$ (Fig. 1A and B). The fluorescent images showed that a large amount of QDs could be observed in J774A.1 cells while only a few QDs were present in Hepa 1-6 cells, which might be due to the innate ability of macrophages to take up the foreign particles for clearance (Fig. 2A). We then enumerated the percentage of cells with QD localization, and quantified the intensity of QD fluorescence in both types of cells using the software Image-Pro-Plus (Media Cybernetics, Inc. USA). As shown in Fig. 2A, the percentage of QD-positive 


\section{(A)}

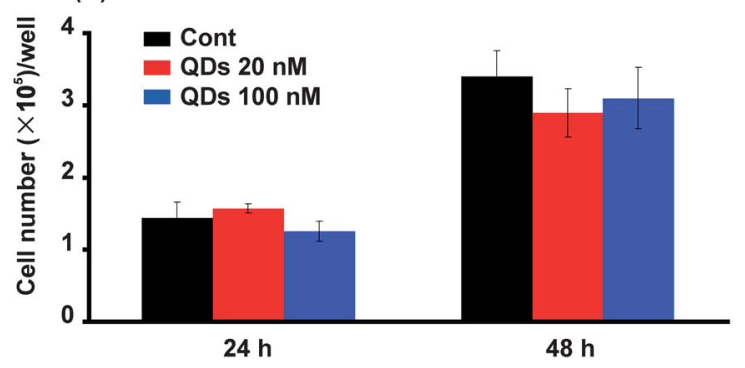

(B)

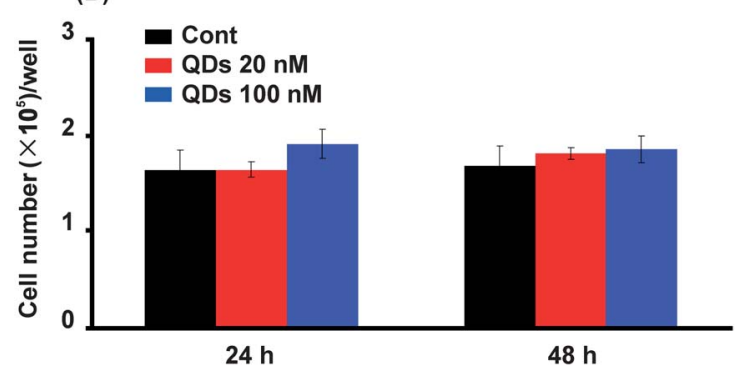

Fig. 1 The number of J774A.1 cells (A) and Hepa 1-6 cells (B) were counted after $24 \mathrm{~h}$ and $48 \mathrm{~h}$ exposure to QDs at 20 and $100 \mathrm{nM}$.

J774A. 1 cells was much higher than that of Hepa $1-6$ cells $(70 \%$ vs. $7 \%, P<0.001)$; the intensity of QD fluorescence in J774A.1 cells was much greater than that of Hepa $1-6$ cells $(>17$ fold, $P<0.001)$.
Although previous data suggested Kuppfer cells rather than hepatocytes might have greater ability in retaining QD particles, ${ }^{12}$ our results from the co-culture system, for the first time, provide direct evidence demonstrating the quantitatively distinct capacity between macrophages and hepatocytes in engulfing and accumulating QDs.

In order to depict precisely the intracellular localization of QDs in J774A.1 cells, the technique of confocal laser scanning microscopy was used to assess the distribution pattern of QD particles among the nucleus, cytoplasm and membrane. We first evaluated the cytotoxicity of QDs to J774A.1 cells. Our data suggest that QDs do not adversely affect cell growth or the survival of J774A.1 cells at a concentration lower than $50 \mathrm{nM}$ (Fig. 1). We stained the nuclei with 4',6-diamidino-2-phenylindole (DAPI, blue) and the cytoskeleton with FITC-conjugated phalloidin (green). The fluorescence for the nuclei, cytoskeleton and QDs were assessed through confocal laser scanning microscopy. As shown in Fig. 2B, upon the $20 \mathrm{nM}$ QD exposure, the majority of QDs (red staining) were localized into the cytoplasm, and only a few QDs were observed in the nuclei (blue). On the one hand, due to their superfine size, QDs might have the capability to enter cells via fluid-phase endocytosis, and remain within the endocytic pathway. ${ }^{13,14}$ On the other hand, monocytes/macrophages are pivotal constituents of cells that engulf foreign particles via phagocytosis. Thus, it could be assumed that J774A.1 macrophages ingest QDs via both fluid-phase endocytosis and phagocytosis.

Macrophages are the most vital type of cells within the innate immune system, and takes a central role in recognizing and eliminating microbial pathogens and foreign particles. Furthermore,
(A)
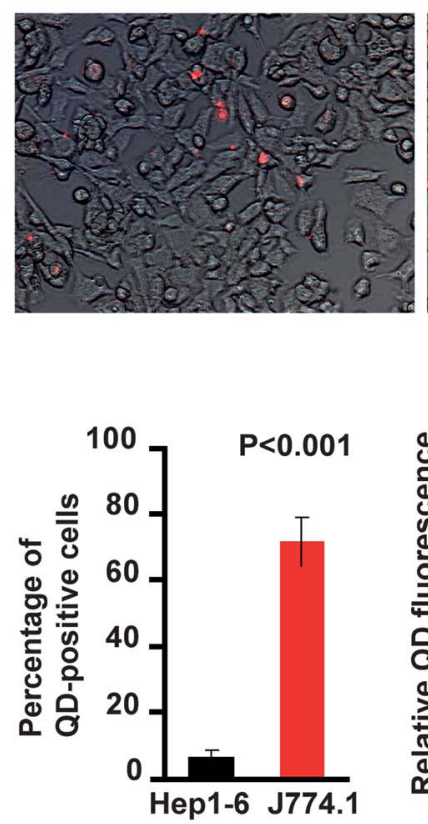

J774.1 macrophages
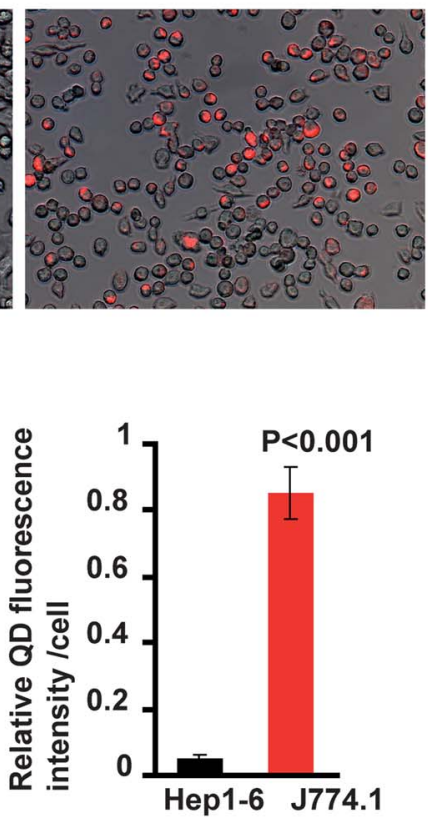

Co-culture of both

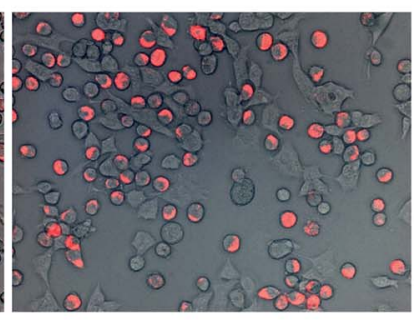

(B)

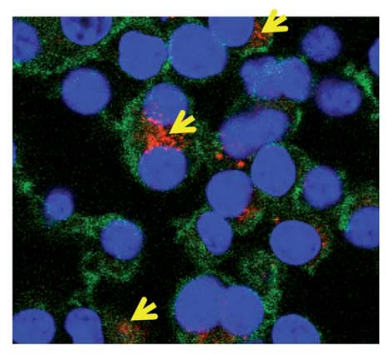

Fig. 2 The preferential localization of QDs in macrophages. (A) The individual culture and co-culture of Hepa 1-6 and J774A.1 cells upon exposure of $20 \mathrm{nM}$ QDs for $24 \mathrm{~h}$. The fluorescence of QDs was visualized under a fluorescent microscope. The image for QD fluorescence was merged into its corresponding bright-contrast image. Original magnification, $\times 200$. The bar graphs show the percentage of QD-positive cells and the intensity of QD fluorescence in J774A. 1 and Hepa 1-6 cells. The results were presented as mean \pm SD. Regarding the enumeration of the percentage of QD-positive cells, there were 3 replicates $(n=3)$. With respect to the intensity of QD fluorescence, $40-50$ cells $(n=40-50)$ from the 3 replicates were examined. (B) The subcellular localization of QDs. Cells upon $20 \mathrm{nM}$ QD treatment for $24 \mathrm{~h}$ were co-stained with DAPI and FITC-conjugated phalloidin. The fluorescence for DAPI, FITC and QDs was assessed through confocal laser scanning microscopy. The cytoskeleton was stained in green, and the nuclei were stained in blue. QDs were visualized in red. The images of the 3 colors were merged together. The arrows indicate QDs located in cytoplasm. Original magnification, $\times 400$. 
macrophages control iron recycling via ingression of iron from hemoglobin of senescent red blood cells (RBCs) and egression of iron into serum through ferroportin for erythropoiesis and other biological processes. To determine whether the deposition of QDs inside macrophages would cause impairment to macrophagic function, we then assessed erythrophagocytosis in ingesting senescent RBCs by J774A.1 cells in vitro upon QD exposure. The aging of RBCs was induced by elevating the intracellular calcium concentration, and only senescent RBCs could be ingested by macrophages. As shown in Fig. $3 \mathrm{~A}, 65 \%$ of the macrophages in the blank control contained ingested RBCs, while $38 \%$ of the macrophages held RBCs upon exposure to $20 \mathrm{nM}$ QDs $(P<0.05)$. The decrease of RBC-laden J774A.1 cells upon exposure to QDs indicates that the accumulation of QD particles inside macrophages reduces their ability in cleaning aged RBCs, and consequently in modulating iron recycling. To examine whether QDs cause impaired erythrophagocytosis in vivo, we established a mouse model of QD exposure at $200 \mathrm{nM}$ for 12 days. Mice developed severe splenomegaly upon QD exposure; the weight of spleen was enlarged by approximately $40 \%$ compared to the PBS control mice $(P<0.05)$ (Fig. 3B). We postulated that the splenic enlargement was the result of $\mathrm{RBC}$ retention in the spleen caused by impaired erythrophagocytosis. As expected, histological examination revealed a substantial increase in RBCs among splenocytes from mice treated with $200 \mathrm{nM}$ QDs in comparison to the control mice (Fig. 3C). The liver weight was not significantly changed in mice with QD treatment compared to the control mice (data not shown). Senescent RBCs are captured and endocytosed by macrophages in various organs, of which the spleen is a major one. If the ability of phagocytosis of macrophages is attenuated, senescent RBCs will be largely retained in the spleen. ${ }^{15}$ Therefore, we observed RBC retention in the spleen and severe splenomegaly due to weakened erythrophagocytosis in response to chronic exposure to QDs.

Our recent study also suggested that QD exposure caused significant morphological changes to macrophages, as J774A.1 cells became round and condensed with fewer surface protrusions in response to QD treatment. ${ }^{6}$ Moreover, we demonstrated here that QDs' intracellular localization undermined the capability of macrophagic phagocytosis. We therefore investigated the molecular basis underlying QD-induced impairment to macrophagic morphology and phagocytosis. Since actin is fundamental to cellular

\section{(A)}
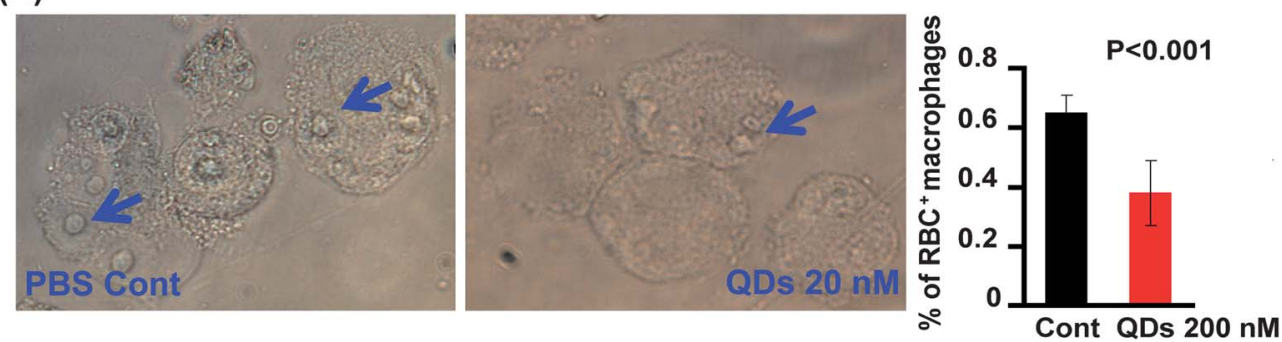

(B)
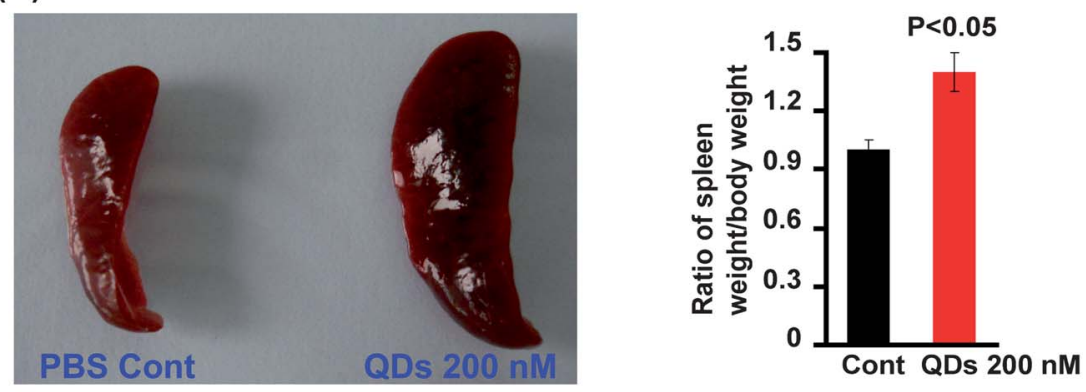

(C) PBS Cont
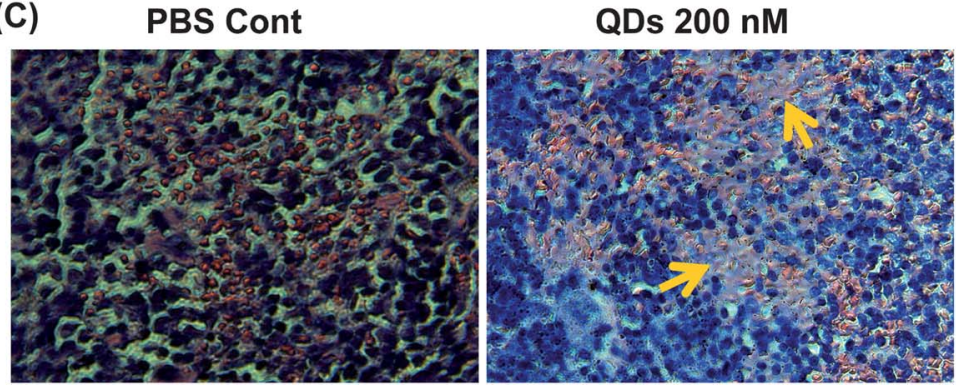

Fig. 3 Intracellular QDs impaired macrophagic erythrophagocytosis in vitro and in vivo. (A) The representative images of erythrophagocytosis performed by J774A. 1 cells in vitro with or without QD treatment at $20 \mathrm{nM}$ for $24 \mathrm{~h}$. Blue arrows indicate ingested RBCs in macrophages. The percentage of RBC-laden J774A.1 cells was quantified (the bar graph). The results are presented as mean $\pm \mathrm{SD}(n=8-10)$. (B) The representative images of spleens from mice treated with $200 \mathrm{nM}$ QDs or PBS for 12 days, respectively. The ratio of spleen weight/body weight was shown in the bar graph. The results are presented as mean $\pm \mathrm{SD}(n=10)$. (C) The representative histological images of spleens from mice with exposure to $200 \mathrm{nM}$ QDs or PBS for 12 days, respectively. The yellow arrows indicate retention of RBCs in the spleen. Original magnification, $\times 400$. 
(A)

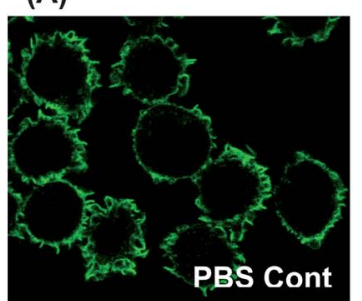

(B)

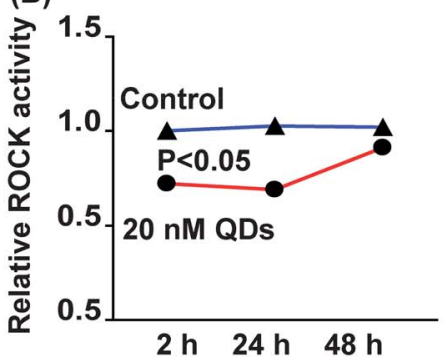

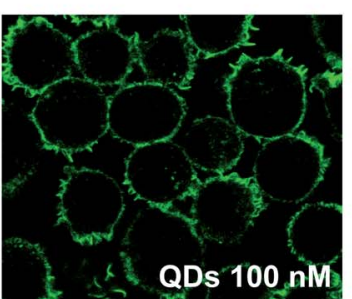

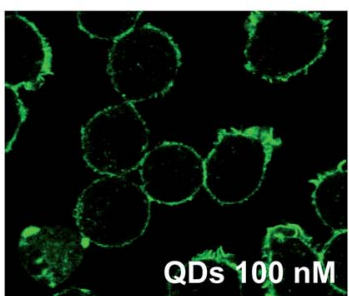

(C)

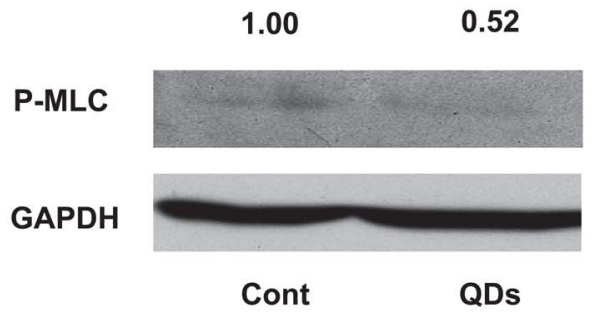

Fig. 4 QDs diminished the ROCK activity. (A) The actin cytoskeleton staining of J774A.1 cells with 20 and $100 \mathrm{nM}$ QD treatment for $24 \mathrm{~h}$. The actin meshwork was stained with FITC-conjugated phalloidin (green). Original magnification, $\times 400$. (B) The ROCK activity in J774A.1 cells upon $20 \mathrm{nM}$ QD treatment for $2 \mathrm{~h}, 24 \mathrm{~h}$ and $48 \mathrm{~h}$. Three replicates $(n=3)$ for each group were assessed. (C) The level of phosphorylated MLC was examined by Western blot analysis for J774A.1 cells upon $20 \mathrm{nM}$ QD treatment for $2 \mathrm{~h}$. The intensities of autoradiogram in Western blots were quantified with the software Image $\mathbf{J}$ (NIH, USA). The quantified data for P-MLC were normalized to those of GAPDH.

morphological changes, we then assessed the density and assembly of actin meshwork by staining the actin fibers with FITC-conjugated phalloidin. As shown in Fig. 4A, there was significant disassembly of filamentous actin (F-actin) and reduction of stress fiber formation, resulting in a reduction in the density of actin meshwork and in the numbers of actin-rich surface protrusions in cells upon exposure to QDs at $20 \mathrm{nM}$, and especially at $100 \mathrm{nM}$, compared to the blank control cells. ROCK is a key determinant of actin polymerization, as ROCK inhibition reduces the F-actin/globular-actin (G-actin) ratio and consequently impairs cytoskeletal meshwork and actin-rich structures (such as macrophagic surface protrusions and phagosomes). ${ }^{16,17}$ We therefore assayed the ROCK activity in J774A.1 cells treated with $20 \mathrm{nM}$ QDs. The ROCK activity was reduced by $\sim 30 \%$ quickly at $2 \mathrm{~h}$ and similarly at $24 \mathrm{~h}$ upon QD treatment; the reduction lasted up to $48 \mathrm{~h}(P<0.05$, Fig. 4B). ROCK controls cellular architecture and force generation principally by phosphorylating the myosin light chain (MLC), and phosphorylation of MLC is

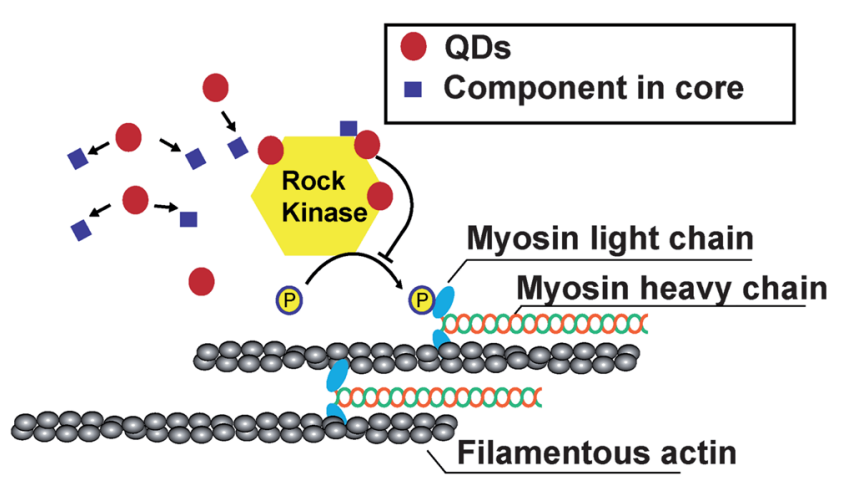

Fig. 5 A schematic describing the mechanism responsible for QDinduced impairment to macrophagic morphology and function. the key event for actin-myosin-based contraction, enhancing the assembly of filamentous myosin heavy chains and increasing the binding of myosin and F-actin. ${ }^{18,19}$ As expected, we observed a large reduction of phosphorylated MLC (P-MLC) upon QD exposure in J774A.1 cells (Fig. 4C). Additionally, remodeling of actin cytoskeleton is a prerequisite for the phagocytic process, as blockade of actin polymerization inhibits erythrophagocytosis by murine macrophages. ${ }^{20}$ ROCK is involved in the phagocytosis process by regulating the actin cytoskeletal remodeling, and diminished ROCK activities would weaken actin remodeling, and impair the formation of actin-dependent phagosome. ${ }^{21,22}$ Thus, QD-caused ROCK attenuation is believed to be responsible for impairments in morphology and erythrophagocytosis for J774A.1 macrophages challenged by the accumulation of QD particles.

QDs display great promise in imaging for diseases (such as cancers and heart disease) due to their novel characteristics in fluorescence, e.g. wide excitation spectra with narrow emission peaks, long life times, and great resistance to photo breaching. In addition, the strong brightness of QDs offers high sensitivity for detection and imaging. The combination of these features endows QDs with unique advantages in imaging and labelling. However, the side and adverse effects of QDs are also of great concern. The mechanism underlying QD-induced cytotoxicity might result from QD particles per se and cadmium ions liberated from the QD core as well. ${ }^{5}$ In the current study, we demonstrated that macrophages are the prominent cells to engulf QDs, and endocytosed QDs mainly stay in the cytoplasm. We further described a novel toxicity to macrophages because of QDs' intracellular accumulation: QD-laden macrophages display significant reduction in erythrophagocytosis both in vitro and in vivo. Furthermore, our results suggest that QDs impair the fidelity of the actin cytoskeleton and actin-rich structures by diminishing the ROCK signalling, causing blunted macrophagic morphology and reduced ability to phagocytize (Fig. 5). 


\section{Conclusions}

Taken together, our data offer insights into the localization of QDs within macrophages and causal impairment to macrophagic function: the consequential damage to actin meshwork, coupled with QDs'-mediated inhibition on the ROCK signalling, curbs the macrophagic ability in erythrophagocytosis and suppresses the recycling of RBCs with consequent splenic $\mathrm{RBC}$ retention in animals. These findings will contribute to obtaining a better understanding of QDs' cellular uptake, intracellular trafficking and biological fate.

\section{Acknowledgements}

This work was supported by grants from the Chinese Academy of Sciences (KZCX2-EW-404), and National Natural Science Foundation of China (grant numbers: 21077128, 20921063, 21177151).

\section{References}

1 S. T. Selvan, T. T. Tan, D. K. Yi and N. R. Jana, Langmuir, 2010, 26, 11631-11641.

2 M. Bruchez, Jr, M. Moronne, P. Gin, S. Weiss and A. P. Alivisatos, Science, 1998, 281, 2013-2016.

3 R. J. Byers and E. R. Hitchman, Prog. Histochem. Cytochem., 2011, 45, 201-237.

4 A. Nel, T. Xia, L. Madler and N. Li, Science, 2006, 311, 622627.

5 R. Hardman, Environ. Health Perspect., 2006, 114, 165-172.
6 W. Liu, S. P. Zhang, L. X. Wang, C. Qu, C. W. Zhang, L. Hong, L. Yuan, Z. H. Huang, Z. Wang, S. J. Liu and G. B. Jiang, PLoS One, 2011, 6, e24406.

7 S. J. Liu, R. N. Suragani, A. Han, W. Zhao, N. C. Andrews and J. J. Chen, Haematologica, 2008, 93, 753-756.

8 S. J. Liu, R. H. Goldstein, E. M. Scepansky and M. Rosenblatt, Cancer Res., 2009, 69, 8742-8751.

9 M. Fernandez-Suarez and A. Y. Ting, Nat. Rev. Mol. Cell Biol., 2008, 9, 929-943.

10 S. J. Liu, R. N. Suragani, F. Wang, A. Han, W. Zhao, N. C. Andrews and J. J. Chen, J. Clin. Invest., 2007, 117, 3296-3305.

11 B. E. Reubinoff, M. F. Pera, C. Y. Fong, A. Trounson and A. Bongso, Nat. Biotechnol., 2000, 18, 559-559.

12 R. S. Yang, L. W. Chang, J. P. Wu, M. H. Tsai, H. J. Wang, Y. C. Kuo, T. K. Yeh, C. S. Yang and P. Lin, Environ. Health Perspect., 2007, 115, 1339-1343.

13 R. Kikkeri, B. Lepenies, A. Adibekian, P. Laurino and P. H. Seeberger, J. Am. Chem. Soc., 2009, 131, 2110-2112.

14 A. Anas, H. Akita, H. Harashima, T. Itoh, M. Ishikawa and V. Biju, J. Phys. Chem. B, 2008, 112, 10005-10011.

15 T. Ishikawa-Sekigami, Y. Kaneko, H. Okazawa, T. Tomizawa, J. Okajo, Y. Saito, C. Okuzawa, M. Sugawara-Yokoo, L. Nishiyama, H. Ohnishi, T. Matozaki and Y. Nojima, Blood, 2006, 107, 341-348.

16 C. L. Lau, R. D. O'Shea, B. V. Broberg, L. Bischof and P. M. Beart, Br. J. Pharmacol., 2011, 163, 533-545.

17 C. H. Seo, K. Furukawa, K. Montagne, H. Jeong and T. Ushida, Biomaterials, 2011, 32, 9568-9575.

18 M. Vicente-Manzanares, X. Ma, R. S. Adelstein and A. R. Horwitz, Nat. Rev. Mol. Cell Biol., 2009, 10, 778-790.

19 S. J. Liu, Mol. Biol. Rep., 2010, 38, 1363-1366.

20 G. Kaplan, Scand. J. Immunol., 1977, 6, 797-807.

21 R. C. May and L. M. Machesky, J. Cell Sci., 2001, 114, 1061-1077.

22 F. Castellano, P. Chavrier and E. Caron, Semin. Immunol., 2001, 13, 347-355. 